\title{
THE EXISTENCE OF POSITIVE SOLUTIONS FOR AN ELLIPTIC BOUNDARY VALUE PROBLEM
}

\author{
G. A. AFROUZI
}

Received 28 November 2004 and in revised form 4 July 2005

By using the mountain pass lemma, we study the existence of positive solutions for the equation $-\Delta u(x)=\lambda(u|u|+u)(x)$ for $x \in \Omega$ together with Dirichlet boundary conditions and show that for every $\lambda<\lambda_{1}$, where $\lambda_{1}$ is the first eigenvalue of $-\Delta u=\lambda u$ in $\Omega$ with the Dirichlet boundary conditions, the equation has a positive solution while no positive solution exists for $\lambda \geq \lambda_{1}$.

\section{Introduction}

Consider the boundary value problem

$$
\begin{gathered}
-\Delta u(x)=\lambda(u|u|+u)(x), \quad x \in \Omega, \\
u(x)=0, \quad x \in \partial \Omega
\end{gathered}
$$

where $\Omega$ is a bounded region with smooth boundary in $\mathbb{R}^{N}, \lambda>0$ is a real parameter, and $\Delta$ is the Laplacian operator.

The study of (1.1) is motivated by the fact that the equation has wide applications to physical models (see [4] and the references therein), our analysis is based on a method used by Rabinowitz [6] and also by Alama and Del Pino [2].

It is well known that steady-state solutions of

$$
\begin{gathered}
-\Delta u(x)=\lambda f(x, u(x)), \quad x \in \Omega, \\
u(x)=0, \quad x \in \partial \Omega,
\end{gathered}
$$

correspond to critical points of the functional $J_{\lambda}: W_{0}^{1,2}(\Omega) \rightarrow \mathbb{R}$,

$$
J_{\lambda}(u)=\frac{1}{2} \int_{\Omega}|\nabla u(x)|^{2} d x-\int_{\Omega} F(x, u(x)) d x,
$$

where $F(x, u)=\int_{0}^{u} f(x, s) d s$. For convenience, we will denote $W_{0}^{1,2}$ by $X$ and will use the 
standard norm

$$
\|u\|_{X}^{2}=\int_{\Omega}|\nabla u(x)|^{2} d x
$$

(see, e.g., [1] or [5]). Henceforth, we will assume that, unless otherwise stated, integrals are over $\Omega$. When $J$ is bounded below on $X, J$ has a minimizer on $X$ which is a critical point of $J$. In many problems such as (1.1), $J$ is not bounded below on $X$, however, in such cases, we may be able to use mountain pass lemma. The mountain pass lemma was introduced by Ambrosetti and Rabinowitz in 1973.

Lemma 1.1 (mountain pass lemma [3]). Let E be a Banach space over $\mathbb{R}$. Let $B_{r}=\{u \in E$ : $\|u\|<r\}$ and $S_{r}=\partial B_{r} ; B_{1}$ and $S_{1}$ will be denoted by $B$ and $S$, respectively. Let $I \in C^{1}(E, \mathbb{R})$. If I satisfies $I(0)=0$ and

(I1) there exist $\rho>0$ and $\alpha>0$, such that $I>0$ in $B_{\rho}-\{0\}$ and

$$
I \geq \alpha>0
$$

on $S_{\rho}$;

(I2) there exists $e \in E, e \neq 0$ with $I(e)=0$;

(I3) if $\left\{u_{m}\right\} \subset E$ with the properties that $I\left(u_{m}\right)$ is bounded above, and $I^{\prime}\left(u_{m}\right) \rightarrow 0$ as $m \rightarrow \infty$, then $\left\{u_{m}\right\}$ possesses a convergent subsequence; and if

$$
\Gamma=\{g \in C([0,1], E): g(0)=0, g(1)=e\} \text {, }
$$

then

$$
b: \equiv \inf _{g \in \Gamma} \max _{y \in[0,1]} I(g(y))
$$

is a critical value of $I$ with $0<\alpha \leq b<+\infty$.

\section{Main results}

The corresponding Euler functional for (1.1) is given by

$$
J_{\lambda}(u)=\frac{1}{2} \int|\nabla u(x)|^{2} d x-\frac{\lambda}{2} \int u^{2}(x) d x-\frac{\lambda}{3} \int|u|^{3}(x) d x .
$$

First, we claim that $J_{\lambda}(u)$ has neither a global minimum nor a global maximum. In fact, we can choose a sequence $\left\{u_{n}\right\}$ satisfying $\int\left|u_{n}\right|^{3} d x=1$ and $\int\left|\nabla u_{n}\right|^{2} d x \rightarrow+\infty$, so that $J_{\lambda}\left(u_{n}\right) \rightarrow+\infty$ as $n \rightarrow+\infty$, that is, $J_{\lambda}(u)$ is not bounded from above. On the other hand, for fixed $u \neq 0$, we have

$$
\begin{aligned}
J_{\lambda}(t u) & =\frac{1}{2} t^{2} \int|\nabla u(x)|^{2} d x-\frac{\lambda}{2} t^{2} \int u^{2}(x) d x-\frac{\lambda}{3}|t|^{3} \int|u|^{3}(x) d x \\
& =t^{2}\left[\frac{1}{2} \int|\nabla u|^{2} d x-\frac{\lambda}{2} \int u^{2}(x) d x-\frac{\lambda}{3}|t| \int|u|^{3}(x) d x\right] \rightarrow-\infty
\end{aligned}
$$

as $t \rightarrow+\infty$. Hence, $J_{\lambda}(u)$ is not bounded from below, so we have provedthe following. 
THEOREM 2.1. $J_{\lambda}(u)$ is neither bounded from above nor bounded from below.

We now show that the mountain pass lemma can be applied in this case.

Theorem 2.2. For every $\lambda<\lambda_{1}$, the problem (1.1) has a positive solution.

Proof. For the existence of solution to (1.1), it is sufficient to check that conditions (I1), (I2), and (I3) of mountain pass lemma are satisfied.

(I1) By the Sobolev imbedding inequality $\left(\int|u|^{3} d x\right)^{1 / 3} \leq c\left(\int|\nabla u|^{2} d x\right)^{1 / 2}(c$ is a positive constant independent of $u \in X)$, we have

$$
\begin{aligned}
J_{\lambda}(u) & =\frac{1}{2} \int|\nabla u|^{2} d x-\frac{\lambda}{2} \int u^{2} d x-\frac{\lambda}{3} \int|u|^{3} d x \\
& \geq \frac{1}{2} \int|\nabla u|^{2} d x-\frac{\lambda}{2 \lambda_{1}} \int|\nabla u|^{2} d x-\frac{\lambda}{3} c^{3}\left(\int|\nabla u|^{2} d x\right)^{3 / 2} \\
& =\left[\frac{1}{2}-\frac{\lambda}{2 \lambda_{1}}-\frac{\lambda}{3} c^{3}\|u\|\right] \int|\nabla u|^{2} d x .
\end{aligned}
$$

So by choosing $\rho=\left(\lambda_{1}-\lambda\right) / \lambda \lambda_{1} c^{3}$ and $\alpha=\left(\lambda_{1}-\lambda\right)^{3} / 6 \lambda^{2} \lambda_{1}^{3} c^{6}$, we will have $J_{\lambda}(u)>0$ for $u \in\{u \in X:\|u\|<\rho\}-\{0\}, J_{\lambda}(u) \geq \alpha$ for $\|u\|=\rho$. Hence, $J_{\lambda}(u)$ satisfies (I1).

(I2) For given fixed $u>0$ in $X$, we consider the map $t \rightarrow J_{\lambda}(t u)$. Since $\lambda<\lambda_{1}$, using the Poincaré inequality, we have $\int|\nabla u|^{2} d x-\lambda \int u^{2} \geq \lambda_{1} \int u^{2}-\lambda \int u^{2}=\left(\lambda_{1}-\lambda\right) \int u^{2}>0$. Then for $t>0$, we have

$$
J_{\lambda}(t u)=t^{2}\left[\frac{1}{2} \int|\nabla u|^{2} d x-\frac{1}{2} \lambda \int u^{2} d x-\frac{1}{3} \lambda|t| \int|u|^{3} d x\right]
$$

which is negative if $t$ is large enough and is positive for sufficiently small $t$. Thus by continuity, there exists $t_{0}>0$ such that $J_{\lambda}\left(t_{0} u\right)=0$, and so $J_{\lambda}(u)$ satisfies (I2).

(I3) We take a sequence $\left\{u_{n}\right\} \subset X$ satisfying $J_{\lambda}\left(u_{n}\right)<M$ (M is a positive constant) and $J_{\lambda}^{\prime}\left(u_{n}\right) \rightarrow 0$ in $X($ as $n \rightarrow \infty)$. Thus, there exists $N$ such that

$$
-\|v\| \leq<J_{\lambda}^{\prime}\left(u_{n}\right), \quad v>=\int \nabla u_{n} \nabla v d x-\lambda \int u_{n} v-\lambda \int u_{n}\left|u_{n}\right| v d x \leq\|v\|
$$

for $n>N$ and $v \in X$. Setting $v=u_{n}$, we have

$$
-\left\|u_{n}\right\| \leq<J_{\lambda}^{\prime}\left(u_{n}\right), \quad u_{n}>=\int\left|\nabla u_{n}\right|^{2} d x-\lambda \int u_{n}^{2} d x-\lambda \int\left|u_{n}\right|^{3} d x \leq\left\|u_{n}\right\|
$$

that is,

$$
-\left\|u_{n}\right\|-\left\|u_{n}\right\|^{2} \leq-\lambda \int u_{n}^{2} d x-\lambda \int\left|u_{n}\right|^{3} d x \leq\left\|u_{n}\right\|-\left\|u_{n}\right\|^{2}
$$


for $n>N$. Also we have

$$
\begin{aligned}
M>J_{\lambda}\left(u_{n}\right) & =\frac{1}{2} \int\left|\nabla u_{n}\right|^{2} d x-\frac{\lambda}{2} \int u_{n}^{2} d x-\frac{\lambda}{3} \int\left|u_{n}\right|^{3} d x \\
& =\frac{1}{2} \int\left|\nabla u_{n}\right|^{2} d x+\frac{1}{3}\left[-\lambda \int u_{n}^{2} d x-\lambda \int\left|u_{n}\right|^{3} d x\right]-\frac{\lambda}{6} \int u_{n}^{2} d x \\
& \geq \frac{1}{2}\left\|u_{n}\right\|^{2}+\frac{1}{3}\left[-\left\|u_{n}\right\|-\left\|u_{n}\right\|^{2}\right]-\frac{\lambda}{6} \int u_{n}^{2} d x \\
& =\frac{1}{6}\left\|u_{n}\right\|^{2}-\frac{1}{3}\left\|u_{n}\right\|-\frac{\lambda}{6} \int u_{n}^{2} d x \\
& \geq \frac{1}{6}\left\|u_{n}\right\|^{2}-\frac{1}{3}\left\|u_{n}\right\|-\frac{\lambda}{6 \lambda_{1}}\left\|u_{n}\right\|^{2} \\
& =\frac{1}{6}\left(1-\frac{\lambda}{\lambda_{1}}\right)\left\|u_{n}\right\|^{2}-\frac{1}{3}\left\|u_{n}\right\|
\end{aligned}
$$

and since $\lambda<\lambda_{1}$, it follows that $\left\{u_{n}\right\}$ is bounded in $X$. Hence, there exists a subsequence, again denoted by $\left\{u_{n}\right\}$, satisfying $u_{n} \rightarrow u_{0}$ weakly in $X$ and strongly in $L^{2}(\Omega)$ and $L^{3}(\Omega)$. Since $J_{\lambda}^{\prime}\left(u_{n}\right) \rightarrow 0$, we have

$$
\begin{aligned}
\left\langle J_{\lambda}^{\prime}\left(u_{n}\right)-J_{\lambda}^{\prime}\left(u_{0}\right), u_{n}-u_{0}\right\rangle= & J_{\lambda}^{\prime}\left(u_{n}\right)\left(u_{n}-u_{0}\right)-J_{\lambda}^{\prime}\left(u_{0}\right)\left(u_{n}-u_{0}\right) \\
= & \int\left(\nabla u_{n}-\nabla u_{0}\right) \nabla\left(u_{n}-u_{0}\right) d x-\lambda \int\left(u_{n}-u_{0}\right)\left(u_{n}-u_{0}\right) d x \\
& -\lambda \int\left(u_{n}\left|u_{n}\right|-u_{0}\left|u_{0}\right|\right)\left(u_{n}-u_{0}\right) d x \longrightarrow 0
\end{aligned}
$$

as $n \rightarrow \infty$. Since $u_{n} \rightarrow u_{0}$ in $L^{2}(\Omega), \int\left(u_{n}-u_{0}\right)^{2} d x \rightarrow 0$ as $n \rightarrow \infty$, also by Holder's inequality, we have

$$
\begin{aligned}
\left|\int\left(u_{n}\left|u_{n}\right|-u_{0}\left|u_{0}\right|\right)\left(u_{n}-u_{0}\right) d x\right| \leq & \left|\int u_{n}\right| u_{n}\left|\left(u_{n}-u_{0}\right) d x\right|+\left|\int u_{0}\right| u_{0}\left|\left(u_{n}-u_{0}\right) d x\right| \\
\leq & \left(\int u_{n}^{3} d x\right)^{2 / 3}\left(\int\left(u_{n}-u_{0}\right)^{3} d x\right)^{1 / 3} \\
& +\left(\int u_{0}^{3} d x\right)^{2 / 3}\left(\int\left(u_{n}-u_{0}\right)^{3} d x\right)^{1 / 3} \rightarrow 0
\end{aligned}
$$

as $u_{n} \rightarrow u_{0}$ in $L^{3}(\Omega)$, and so $\int\left(u_{n}\left|u_{n}\right|-u_{0}\left|u_{0}\right|\right)\left(u_{n}-u_{0}\right) d x \rightarrow 0$. Thus,

$$
\int\left(\nabla u_{n}-\nabla u_{0}\right) \nabla\left(u_{n}-u_{0}\right) d x \longrightarrow 0
$$

and so $\int\left|\nabla u_{n}\right|^{2} d x \rightarrow \int\left|\nabla u_{0}\right|^{2} d x$. This, together with the weak convergence of $\left\{u_{n}\right\}$, implies that $\left\{u_{n}\right\}$ is convergent strongly in $X$. Hence, $J_{\lambda}(u)$ satisfies the condition (I3). Therefore, by the mountain pass lemma, $J_{\lambda}(u)$ has a nontrivial critical point denoted by $u_{*}$. Since $J_{\lambda}\left(u_{*}\right)=J_{\lambda}\left(\left|u_{*}\right|\right)$, without loss of generality, we may assume that $u_{*}$ is a nonnegative weak solution of (1.1) and it follows from standard regularity arguments that 
$u_{*} \in C^{2}(\Omega)$ is a classical solution of (1.1), that is, we have

$$
\begin{gathered}
-\Delta u_{*}(x)=\lambda\left(u_{*}\left|u_{*}\right|+u_{*}\right)(x), \quad x \in \Omega, \\
u_{*}(x)=0, \quad x \in \partial \Omega,
\end{gathered}
$$

also it is easy to deduce from the maximum principle that $u_{*}>0$ on $\Omega$.

We now show that the problem (1.1) has no positive solution in the case where $\lambda \geq \lambda_{1}$. Theorem 2.3. The problem (1.1) has no a positive solution when $\lambda \geq \lambda_{1}$.

Proof. Let $\lambda \geq \lambda_{1}$ be arbitrary and fixed. On the contrary, we assume that $u$ is a positive solution of (1.1), that is,

$$
\begin{gathered}
-\Delta u(x)=\lambda(u|u|+u)(x), \quad x \in \Omega \\
u(x)=0, \quad x \in \partial \Omega .
\end{gathered}
$$

Let $\phi>0$ be the principal eigenfunction corresponding to principal eigenvalue $\lambda_{1}$, that is,

$$
\begin{gathered}
-\Delta \phi(x)=\lambda_{1} \phi(x), \quad x \in \Omega, \\
\phi(x)=0, \quad x \in \partial \Omega .
\end{gathered}
$$

Multiplying (2.13) by $\phi,(2.14)$ by $u$, and integrating over $\Omega$ give, respectively,

$$
\begin{gathered}
\int \nabla u \nabla \phi d x=\lambda \int u|u| \phi d x+\lambda \int u \phi d x, \\
\int \nabla u \nabla \phi d x=\lambda_{1} \int u \phi d x .
\end{gathered}
$$

By subtracting (2.16) from (2.15), we obtain

$$
0=\left(\lambda-\lambda_{1}\right) \int u \phi d x+\lambda \int u|u| \phi d x
$$

and this is a contradiction as $u$ and $\phi$ are positive and $\lambda \geq \lambda_{1}$, and so the problem (1.1) has no positive solution when $\lambda \geq \lambda_{1}$.

We now will show that when $\lambda$ is sufficiently small, then the positive solution $u$ of (1.1) corresponding to $\lambda$ is very large.

Theorem 2.4. If $\lambda \rightarrow 0$ and $u$ is the positive solution of (1.1) corresponding to $\lambda$, then $\|u\| \rightarrow$ $+\infty$.

Proof. Since $u$ is the positive solution of (1.1) corresponding to $\lambda$, we have

$$
\begin{gathered}
-\Delta u(x)=\lambda(u|u|+u)(x), \quad x \in \Omega, \\
u(x)=0, \quad x \in \partial \Omega .
\end{gathered}
$$

Multiplying (2.18) by $u$ and integrating over $\Omega$ give

$$
\int|\nabla u|^{2} d x=\lambda \int|u|^{3} d x+\lambda \int u^{2} d x
$$


Substituting the Sobolev inequalities

$$
\int|u|^{3} d x \leq c\left(\int|\nabla u|^{2} d x\right)^{3 / 2}, \quad \int|u|^{2} d x \leq \frac{1}{\lambda_{1}} \int|\nabla u|^{2} d x
$$

into (2.19) yields

$$
\int|\nabla u|^{2} d x \leq c \lambda\left(\int|\nabla u|^{2} d x\right)^{3 / 2}+\frac{\lambda}{\lambda_{1}} \int|\nabla u|^{2} d x
$$

Hence,

$$
\left(1-\frac{\lambda}{\lambda_{1}}\right)\|u\|^{2} \leq c \lambda\|u\|^{3}
$$

and so

$$
\|u\| \geq \frac{\lambda_{1}-\lambda}{c \lambda \lambda_{1}}=\frac{1}{c}\left(\frac{1}{\lambda}-\frac{1}{\lambda_{1}}\right) .
$$

Thus $\|u\| \rightarrow+\infty$ as $\lambda \rightarrow 0$.

It is easy to see that $u$ is a positive solution of (1.1) if and only if $u$ is a positive solution of

$$
\begin{gathered}
-\Delta u(x)=\lambda\left(u^{2}+u\right)(x), \quad x \in \Omega, \\
u(x)=0, \quad x \in \partial \Omega,
\end{gathered}
$$

so we have obtained the following theorem.

Theorem 2.5. The problem (2.24) has a positive solution when $\lambda<\lambda_{1}$, while no positive solution exists for $\lambda \geq \lambda_{1}$.

\section{References}

[1] R. A. Adams, Sobolev Spaces, Pure and Applied Mathematics, vol. 65, Academic Press, New York, 1975.

[2] S. Alama and M. Del Pino, Solutions of elliptic equations with indefinite nonlinearities via Morse theory and linking, Ann. Inst. H. Poincaré Anal. Non Linéaire 13 (1996), no. 1, 95-115.

[3] A. Ambrosetti and P. H. Rabinowitz, Dual variational methods in critical point theory and applications, J. Funct. Anal. 14 (1973), 349-381.

[4] F. Gazzola and A. Malchiodi, Some remarks on the equation $-\Delta u=\lambda(1+u)^{p}$ for varying $\lambda$, $p$ and varying domains, Comm. Partial Differential Equations 27 (2002), no. 3-4, 809-845.

[5] D. Gilbarg and N. S. Trudinger, Elliptic Partial Differential Equations of Second Order, Grundlehren der Mathematischen Wissenschaften, vol. 224, Springer, Berlin, 1983.

[6] P. H. Rabinowitz, Minimax Methods in Critical Point Theory with Applications to Differential Equations, CBMS Regional Conference Series in Mathematics, vol. 65, American Mathematical Society, Rhode Island, 1986.

G. A. Afrouzi: Department of Mathematics, Faculty of Basic Science, University of Mazandaran, Pasdaran Street, P.O. Box 416, 47415 Babolsar, Iran

E-mail address: afrouzi@umz.ac.ir 


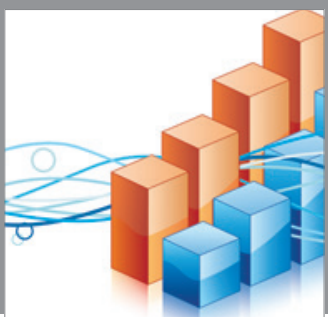

Advances in

Operations Research

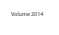

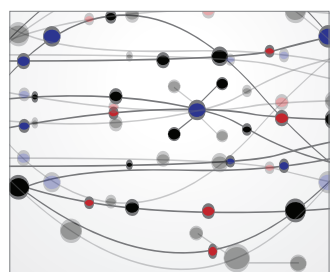

\section{The Scientific} World Journal
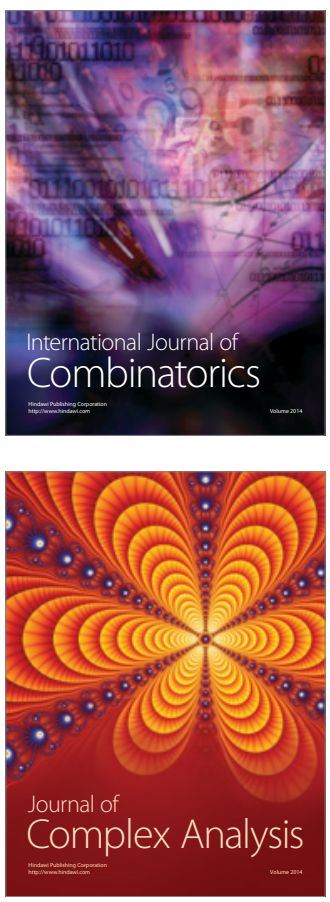

International Journal of

Mathematics and

Mathematical

Sciences
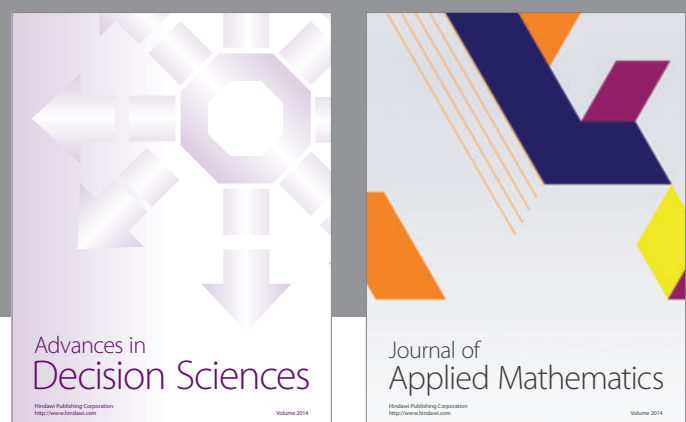

Journal of

Applied Mathematics
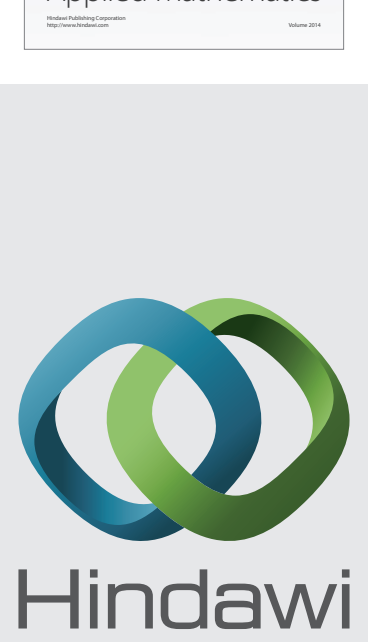

Submit your manuscripts at http://www.hindawi.com
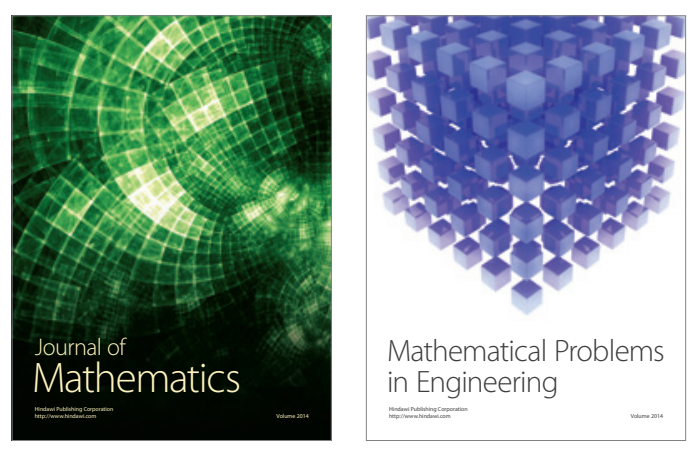

Mathematical Problems in Engineering
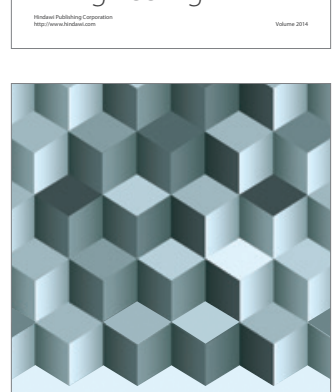

Journal of

Function Spaces
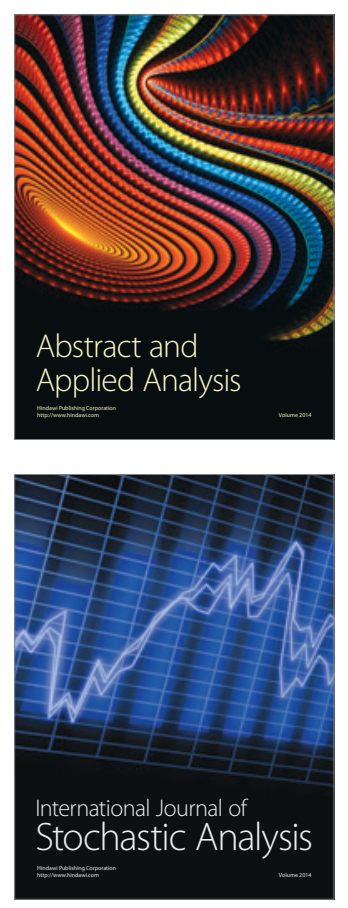

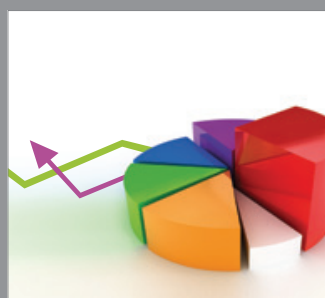

ournal of

Probability and Statistics

Promensencen
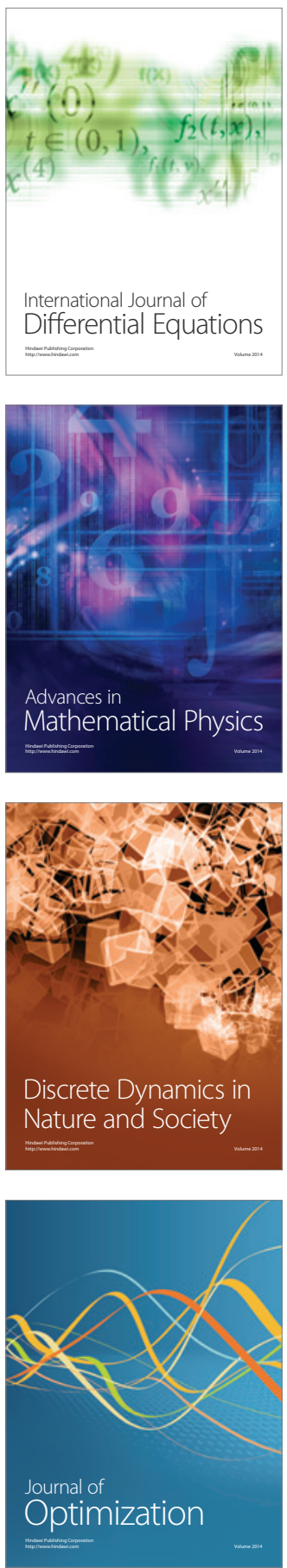